REVISTA CIENCIAS BIOMÉ DICAS

ARTÍCULOS ORIGINALES

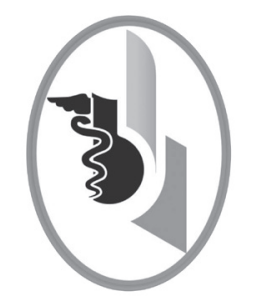

\title{
ENCLAVIJAMIENTO CEFALOMEDULAR EN EL MANEJO DE FRACTURAS INESTABLES DE CADERA
}

\author{
CEPHALOMEDULLARYS FRACTURE FIXATION IN THE \\ MANAGEMENT OF UNSTABLE HIP FRACTURES.
}

Fonseca-Caro John Fredy (1)

Matson-Carballo Gustavo (2)

Pereira-Betancourt Carlos (2)

Rivera-Martínez Edgardo (2)

Correspondencia: johnffc@hotmail.com

Recibido para evaluación: julio - 05 - 2013. Aceptado para publicación: junio - 06 - 2015.

\section{RESUMEN}

Introducción: las fracturas inestables intertrocantéricas de cadera en el adulto mayor son problemas de salud pública. Su manejo se realiza con osteosíntesis con clavos cefalomedulares, de los cuales existen varios diseños.

Objetivos: determinar si hay diferencias en los resultados clínicos con el uso de clavos Targon-PF y hoja espiral TFN.

Metodología: se realizó estudio por conveniencia, prospectivo, en 69 pacientes adultos que presentaron fractura intertrocantérica inestable. Los pacientes fueron llevados a cirugía para osteosíntesis con clavo cefalomedular. Fueron divididos en dos grupos: [A] implante de dos tornillos a cuello (Targon-PF) y [B] Hoja espiral (TFN). Para ambos grupos se realizó técnica quirúrgica convencional y se evaluó el tiempo quirúrgico, sangrado, complicaciones intraoperatorias, resultados de funcionalidad y consolidación clínica y radiológica.

Resultados: se realizó seguimiento promedio de 23 meses. Con el uso de uno u otro implante, no se observaron diferencias estadísticamente significativas en el retorno de la funcionalidad. Fueron más frecuentes las complicaciones Cut Out y mal posición en el grupo Targon-PF, sin diferencia significativa. Fue menor el tiempo quirúrgico con el TFN, $62.4 \pm 15.7$ minutos mientras que con Targon-PF fue $86.5 \pm 31.7$ minutos, $p<0.0002$. Conclusión: no se observaron diferencias significativas en los resultados clínicos con el uso de clavos Targon-PF y hoja espiral TFN. Rev.cienc.biomed. 2015; 6(2):265-271

\section{PALABRAS CLAVES}

Clavos ortopedicos; Fijacion de la fractura; Cadera

1 Médico. Estudiante de postgrado. Ortopedia y Traumatología. Facultad de Medicina. Universidad de Cartagena. Colombia.

2 Médico Ortopedista y Traumatólogo. Universidad de Cartagena. Docente. Facultad de Medicina. Universidad de Cartagena. Clínica Universitaria San Juan de Dios. Cartagena. Colombia. 


\section{SUMMARY}

Introduction: unstable hip fractures in elderly people are a public health problem. The treatment is carried out with osteolysis and cephalomedullarys bone nails of which there are others designs.

Objective: to determine in the clinical results whether there are differences between PF Targon bone nails and TFN spiral plate.

Methods: prospective study was carried out in 69 patients who presented unstable intertrochanteric fracture. The patients were taken to surgery for osteosynthesis with cephalomedullarys bone nails. They were divided in two groups: [A] Two screws fixation in neck (PF Targon) and [B] (TFN) spiral plate. For both groups were performed by conventional surgical technique and evaluated the surgical time, the bleeding, the intraoperative complications, the functionality results, clinical and radiological consolidation.

Results: the procedure was followed in an average of 23 months. No differences were observed in the return of functionality between the use of an implant or other. Complications with Cut Out were more frequent and PF Targon not in the right position without significant difference. Surgical time was lower with TFN, $62.4 \pm 15.7$ minutes, while with Targon-PF was $86.5 \pm 31.7$ minutes $p<0.0002$.

Conclusion: no significant differences were observed in the clinical results between the use of PF Targon bone nails and TFN spiral plate. Rev. cienc.biomed. 2015;6(2):265271

\section{KEY WORDS}

Bone Nails, Fracture Fixation, Hip.

\section{INTRODUCCIÓN}

Las fracturas de cadera son una lesión grave desde el punto de vista social, familiar, económico y de recursos en salud para pacientes de la tercera edad. La incidencia varía de un país a otro, pero se aumenta en países en vía de desarrollo $(1,2,3)$. En los años noventa en Estados Unidos se presentaron en promedio 1.7 millones de fracturas de cadera, de las cuales el $49 \%$ fueron intertrocantéricas. Se estima una incidencia de 63/100 000 habitantes y aumenta con la edad. Estas fracturas se presentan cinco veces más después de los ochenta años de edad $(1,2,4-9)$.

Las lesiones intertrocantéricas por el bajo riesgo de necrosis avascular y su fisiología son candidatas a manejo con osteosíntesis. A través de los tiempos se ha modificado el tipo de implantes con el entendimiento de la biomecánica de la fractura y la búsqueda para obtener mejores resultados funcionales, minimizando las complicaciones quirúrgicas y facilitando la técnica operatoria $(2,4,7-15)$.

En las fracturas intertrocantéricas inestables, la gran conminución y el compromiso de la porción ósea que trasmite las cargas de distención a la diáfisis femoral, suele ser severa. Como primera línea de manejo en esta fractura están los clavos cefalomedulares, que neutralizan dichas cargas y los implantes que presentan ligeras modificaciones para brindar propiedades biomecánicas distintas $(1,4,7,10-12,15)$. Estos últimos intentan minimizar las complicaciones ortopédicas como el Cut Out y la fractura por estrés de la cortical anterior del fémur, así como facilitar la técnica quirúrgica, de manera que se reduzcan la perdida sanguínea, la noxa tisular y el tiempo operatorio, sin perder la rápida movilización y la oportunidad de apoyo $(7,9,16-19)$.

El porcentaje de falla de los implantes en cadera para fracturas intertrocantéricas oscila de 5 al 23\% y la causa más común de falla es el colapso en varo del cuello femoral con Cut Out superior del implante $(12,20)$.

Se ha diseñado en la porción proximal de los implantes un dispositivo que da fijación a través del cuello femoral por medio de una hoja espiral, este tipo de implante se tipificó como TFN (Synthes) (17). En cuanto a los clavos cefalomedulares de última generación con dos tornillos en el cuello de fémur o dis- 
ISSN: 2215-7840, 6(2), julio-diciembre 2015, Fonseca-Caro John Fredy, Matson-Carballo Gustavo, Pereira-Betancourt Carlos,

Rivera-Martínez Edgardo

positivo antirotacional, se caracterizan por incrementar su resistencia a las fuerzas rotacionales no así a las tensionales, ejemplo es el mismo Targon-PF $(12,20)$.

El estudio de Gary (22) es referencia colombiana sobre los resultados de enclavijamiento con clavo cefalomedular de segunda generación. Realizó seguimiento a 25 pacientes y reportó estancia prehospitalaria promedio de doce días, tiempo quirúrgico 55 minutos y sangrado $300 \mathrm{cc}$, pero no estableció seguimiento a largo plazo que permitiese delimitar posibles complicaciones, así mismo, el estudio no permite comparar los resultados funcionales con otro tipo de implantes.

En el manejo de fracturas intertrocantéricas inestables existen dudas en cuanto a si existen diferencias entre un implante u otro, a pesar de tener el mismo principio biomecáni$\mathrm{Co}$, ya que pueden afectar diferentemente el desenlace del paciente y estas diferencias podrían determinar la escogencia de uno u otro implante. Por lo que se comparan los implantes disponibles en la institución, en las variables del acto quirúrgico, en sus resultados funcionales y en las complicaciones a largo plazo. En Cartagena no existen estadísticas sobre estos tipos de fractura, al parecer.

\section{MATERIALES Y MÉTODOS}

Tipo de estudio: serie de casos prospectivocomparativo, muestreo por conveniencia. Se realizó en pacientes con diagnóstico de fractura de cadera intertrocantérica inestable, atendidos en la Clínica Universitaria San Juan de Dios de Cartagena-Colombia, durante el periodo comprendido entre el 1 de julio de 2009 al 31 de diciembre de 2010 y que fueron intervenidos con enclavijamiento cefalomedular.

Los pacientes fueron operados en mesa de fracturas en decúbito supino, se realizó un abordaje lateral por arriba del trocánter mayor y la aplicación de guía intramedular, perfilador y aplicación del implante. Para la fijación a cuello se pasó guía con intensificador y se realizaron pasos según el fabricante para cada implante, la reducción y estabilización se verificó por intensificador de imágenes y la escogencia del implante se dio por seguridad social y contrato con proveedor.
En el intraoperatorio, se valoraron las pérdidas sanguíneas definidas por el cirujano, tiempo quirúrgico, complicaciones intraoperatorias y del implante, el seguimiento clínico se realizó al egreso y de manera mensual por cada paciente, por consolidación radiográfica. Se examinó la presencia de formación de puente óseo en la proyección de radiografía de cadera y consolidación clínica en la ausencia de dolor con apoyo total, la funcionalidad se valoró con la escala de Nurm Marinem modificada (Figura No 1), en la cual se da un punto por cada ítem evaluado de acuerdo a su movilidad cotidiana, siendo el máximo 18 puntos y aplicándose en promedio a los cuatro meses del egreso.

La recolección de datos se realizó mediante el sistema de manejo de historias clínicas habilitado por el programa Hosvital y se recolectaron datos de la descripción quirúrgica, así como del seguimiento intrainstitucional y ambulatorio de los pacientes intervenidos con clavo Targon-PF y TFN, operados por el mismo grupo de especialistas y de asistentes. Dentro del seguimiento prospectivo en la historia se recurrió al Ilamado telefónico del paciente con el fin de determinar su funcionalidad, estado actual y mortalidad.

Los datos se almacenaron en software Excel 2010 y posteriormente se realizó un análisis de tipo descriptivo mediante tabla de frecuencia y medidas de tendencia central y dispersión para variables cuantitativas y cualitativas respectivamente. Las comparaciones entre los grupos se realizaron a través de las pruebas de hipótesis $\mathrm{Chi}^{2}$ o Test de Ficher según fue necesario en variables cualitativas, mientras que en las cuantitativas T student o Mann Winney según criterios de normalidad. Este análisis se apoyará en los programas informáticos Epi-info.

De acuerdo con el Decreto 8430 de 1993 la investigación se realizó sin riesgo debido a que se valora sobre manejo establecido por médico tratante sin intervención sobre el paciente, revisión de historias clínicas y radiografías ya tomadas o aplicadas al paciente y seguimiento observacional o telefónico. Aprobado por el Comité de Ética Médica de la Clínica Universitaria San Juan de Dios Cartagena - Colombia. 


\section{RESULTADOS}

Un total de 69 pacientes participaron en el estudio, de estos nueve fueron excluidos: dos por tener fractura patológica por proceso tumoral, uno por tener politrauma que alteró plan quirúrgico y seis por no tener el seguimiento adecuado. La edad promedio fue de $78.1 \pm 8.2$, sin diferencias entre los dos grupos de implantes y en su mayoría los casos corresponden a mujeres.

El dispositivo Targon-PF se utilizó en fracturas tipo $31 \mathrm{~A} 2$ y $31 \mathrm{~A} 3$ en el $99 \%$ de los casos, y en el dispositivo TFN en el $97 \%$ para el mismo grupo. Por clasificación de tronzo la más común para Targon-PF y TFN fue la IIIB sin diferencias estadísticamente significativas.

En comorbilidades previas a la cirugía, el $70 \%$ de los pacientes sin importar el implante, presentaban comorbilidades previas, la hipertensión en un $41 \%$ en Targon-PF y 50\% en TFN fue la más frecuente. La estancia pre quirúrgica fue en promedio de tres días en ambos implantes (Tabla No 1 ).

El tiempo quirúrgico del clavo Targon-PF fue significativamente más prolongado, $(P<0.0002) 86$ minutos contra 62 en promedio del TFN. El sangrado promedio de pacientes con Targon-PF fue de $100 \mathrm{cc}$ y en los pacientes con TFN $125 \mathrm{cc}$, lo cual no es estadísticamente significativo.

Dentro de los implantes Targon-PF se utilizaron en el $65 \%$ clavos de $180 \mathrm{~mm}$, el más corto de su presentación, y en implantes tipo TFN 67\% clavos de 170 mm, también el más corto de su presentación. En la serie de pacientes no se encontraron complicaciones agudas vistas en series previas como fractura intraoperatoria, sección de trocánter mayor, hematoma o seroma. Se halló presencia de infección en las vías urinarias en el $12.5 \%$ de los pacientes con TargonPF, y en el $5.6 \%$ de los pacientes con TFN; se presentaron escaras en el $8.3 \%$ de pacientes con Targon-PF y en el $16.7 \%$ de pacientes con TFN. Por último, trastorno hidroelectrolítico se encontró en el $20 \%$ de pacientes con clavo tipo Targon-PF y $5.6 \%$ en pacientes con clavo TFN.

De la consolidación clínica solo el $50 \%$ de los pacientes, incluidos ambos implantes, tienen

\begin{tabular}{|c|c|c|c|}
\hline \multicolumn{4}{|c|}{$\begin{array}{l}\text { TABLA No } 1 . \\
\text { CARACTERÍSTICAS SOCIODEMOGRÁFICAS }\end{array}$} \\
\hline & $\begin{array}{c}\text { TARGON- } \\
\text { PF } \\
n: 24\end{array}$ & $\begin{array}{l}\text { TNF } \\
\mathrm{n}: 36\end{array}$ & $P$ \\
\hline Edad $\bar{X} \pm \mathrm{DE}$ & $\begin{array}{c}78.2 \pm \\
8.7\end{array}$ & $\begin{array}{c}77.5 \pm \\
11.7 \\
\end{array}$ & 0.8119 \\
\hline Femenino & $16(66.7)$ & $28(77.8)$ & \multirow{2}{*}{0.5126} \\
\hline Masculino & $8(33.3)$ & $8(22.2)$ & \\
\hline \multicolumn{4}{|c|}{ Lateralidad } \\
\hline Derecho & $11(45.8)$ & $15(41.7)$ & \multirow{2}{*}{0.9617} \\
\hline Izquierdo & $13(54.2)$ & $21(58.3)$ & \\
\hline \multicolumn{4}{|c|}{ Tipo de fractura Clas $\mathrm{AO}$} \\
\hline $31 \mathrm{~A}$ & $0(0.0)$ & $2(5.6)$ & 0.6541 \\
\hline $31 \mathrm{~A} 2$ & $19(79.2)$ & $29(80.6)$ & 0.8440 \\
\hline $31 \mathrm{A3}$ & $5(20.8)$ & $5(17.9)$ & 0.9559 \\
\hline \multicolumn{4}{|c|}{ Tipo de fractura tronzo } \\
\hline I & $0(0.0)$ & $1(2.8)$ & 0.8427 \\
\hline II & $5(20.8)$ & $2(33.3)$ & 0.4469 \\
\hline IIIA & $3(12.5)$ & $4(11.1)$ & 0.8064 \\
\hline IIIB & $10(41.7)$ & $20(27.8)$ & 0.4013 \\
\hline IV & $1(4.2)$ & $4(11.1)$ & 0.6380 \\
\hline $\mathrm{V}$ & $5(20.8)$ & $5(17.9)$ & 0.9559 \\
\hline Comorbilidades & $17(70.8)$ & $25(69.4)$ & 0.8638 \\
\hline HTA & $10(41.7)$ & $18(50.0)$ & 0.7135 \\
\hline Diabetes & $8(33.3)$ & $8(22.2)$ & 0.5126 \\
\hline Coronariopatía & $3(12.5)$ & $4(11.1)$ & 0.8064 \\
\hline EPOC & $2(8.3)$ & $1(2.8)$ & 0.7240 \\
\hline Demencia & $1(4.2)$ & $4(11.1)$ & 0.6380 \\
\hline IRC & $1(4.2)$ & $2(5.6)$ & - \\
\hline $\begin{array}{l}\text { Estancia PreQx } \\
\text { Me }[\mathrm{RI}]\end{array}$ & $3[2-6]$ & $3[2.5-7]$ & 0.2523 \\
\hline
\end{tabular}

registrada la consolidación en la historia clínica. Con seguimiento radiográfico hay nueve pacientes $(15 \%)$, quienes tienen registrada la consolidación pero no tienen fecha exacta de solicitud del mismo en el postoperatorio. La funcionalidad retornó en promedio a un nivel previo al tenido por el paciente, aunque se obtuvo más rápido a nivel óptimo en el enclavijamiento de tipo TFN sin ser estadísticamente significativo.

En las complicaciones tardías no se encontró necrosis avascular, falla de material de osteosíntesis u osificación heterotópica, los dos casos de Cut Out y el caso de mal posición del implante, se encontraron en el gru- 
ISSN: 2215-7840, 6(2), julio-diciembre 2015, Fonseca-Caro John Fredy, Matson-Carballo Gustavo, Pereira-Betancourt Carlos,

Rivera-Martínez Edgardo

po del dispositivo Targon-PF. El dolor anterior de muslo fue frecuente en ambos implantes, cinco pacientes en el caso de Targon-PF y cuatro en el caso de pacientes TFN, lo anterior no guarda relación con el largo del clavo en el estudio.

El seguimiento tardó 23 meses, con un promedio del grupo Targon-PF de 21 meses y del grupo TFN de 25 meses, encontrándose mortalidad general de $22 \%$, siendo $20.8 \%$ en los pacientes con Targon-PF y $25 \%$ en pacientes del grupo TFN. El $71 \%$ de los pacientes que fallecieron tenían estancia prequirúrgica mayor a cinco días y la causa más frecuente de muerte fue neumonía, en $42 \%$ ( $14 \%$ en caso de pacientes con implante tipo Targon-PF y $28 \%$ en TFN), (Tabla No 2 ).

\section{DISCUSIÓN}

El enclavijamiento cefalomedular representa una técnica adecuada para el manejo de fracturas inestables de cadera, es bien utilizada en la institución siguiendo las indicaciones dadas por las casas fabricantes, es decir, fracturas de cadera inestables como AO 31A2, 31 A3 y tronzo IIIB en ambos tipos de implantes.

Los pacientes operados fueron de la tercera edad y en la mayoría de los casos fueron mujeres, quienes se encuentran más propensas a padecer fractura de cadera extracapsular inestable. La presencia de comorbilidades fue en promedio de $50 \%$, muy parecido a la de los trabajos previos a nivel mundial (3, $9,22)$. El promedio de estancia prequirúrgica fue de tres días, la cual es un poco mayor a la descrita por Nikkel (8) en su trabajo sobre el impacto de las comorbilidades en la hospitalización de pacientes con fractura de cadera, en la cual el $84 \%$ de sus pacientes fueron operados en los dos primeros días. Ello es similar en series previas de pacientes, como las descritas por Bucholtz (1), Miyamoto $(3,15)$ y Herrera $(9)$.

El promedio de tiempo quirúrgico fue aceptable para ambos tipos de implantes, siendo menor en el implante TFN dado por la técnica quirúrgica y es muy similar al encontrado en centros de experiencia como el descrito por Nuber en el 2003 (20) y comentado en la revisión sistemática de Miyamoto, Kaplan del
2008 con una serie de 129 pacientes (15). La diferencia de tiempos quirúrgicos fue estadísticamente significativa y representa un valor agregado al dispositivo de hoja espiral TFN con el cual se pueden minimizar las complicaciones inherentes a la anestesia y costos en sala de operaciones. El promedio de sangrado fue de 100 a $125 \mathrm{cc}$, con lo cual no se presenta riesgo de inestabilidad hemodinámica y es mucho menor que el encontrado en trabajos como el de Gary (22).

En series previas como el trabajo de Herrera (9), con seguimiento de 551 pacientes se registraron complicaciones que no se presentaron en nuestro trabajo, tales como sección de trocánter mayor, fractura intraoperatoria, seroma o hematoma. Como complicación se detectó la presencia de escaras y de infección en las vías urinarias, así como trastorno hidroelectrolítico, estas complicaciones se deben minimizar con el mejoramiento del cuidado de enfermería: cambio de posición, medidas antiescaras y manejo de catéteres, las cuales son encontradas en otras series descritas.

Para el seguimiento postoperatorio por parte del Departamento de Ortopedia de la institución donde se realizó el estudio, no se registra de manera adecuada la consolidación clínica ni existen parámetros para seguimiento radiográfico, aunque estos no alteran la funcionalidad clínica o estado del paciente, limita los datos de reparación ósea en este tipo de fracturas y con ello, la determinación del tiempo que el implante soporta, es decir, la totalidad de la carga del paciente.

La funcionalidad del paciente retorna a su estado previo con ambos implantes siendo mejor en el grupo de TFN, con lo cual se busca minimizar las complicaciones propias del encamamiento prolongado. En las complicaciones tardías dicho retorno a la funcionalidad fue similar el reportado en trabajos como el de Lindskog (4), Herrera (9), Nurmi (7). Cinco pacientes de la serie con buena funcionalidad previa no retornaron a la misma y cuatro de estos permanecieron más de siete días en estancia prequirúrgica por comorbilidades múltiples, encontramos el Cut Out y la mal posición del implante en el grupo del implante tipo Targon-PF. En el grupo de investigadores asumimos que sea porque el TAD y las zonas de Cle- 
TABLA NO 2. ESTUDIO INTRAOPERATORIO Y RESULTADOS POSTOPERATORIOS

\begin{tabular}{|l|c|c|c|}
\hline & $\begin{array}{c}\text { TARGON-PF } \\
\mathrm{N}=24\end{array}$ & $\begin{array}{c}\text { TNF } \\
\mathrm{N}=36\end{array}$ & Valor de $\mathrm{p}$ \\
\hline Tiempo quirúrgico $\bar{X}_{ \pm} \mathrm{DE}$ & $86.5 \pm 31.7$ & $62.4 \pm 15.7$ & 0.0002 \\
\hline Sangrado intraoperatorio, Me [RI] & $100[50-100]$ & $125[75-200]$ & 0.6559 \\
\hline Sobredistracción de foco, $\mathrm{n}(\%)$ & $1(4.2)$ & $1(2.8)$ & 0.6626 \\
\hline Infección urinaria, $\mathrm{n}(\%)$ & $3(12.5)$ & $2(5.6)$ & 0.6384 \\
\hline HVDA, $\mathrm{n}(\%)$ & $1(4.2)$ & $1(2.8)$ & 0.6626 \\
\hline Embolismo pulmonar, $\mathrm{n}(\%)$ & $1(4.2)$ & $2(5.6)$ & 0.7193 \\
\hline Escaras, $\mathrm{n}(\%)$ & $2(8.3)$ & $6(16.7)$ & 0.5823 \\
\hline Confusión mental, $\mathrm{n}(\%)$ & $4(16.7)$ & $4(11.1)$ & 0.8123 \\
\hline Evento trombótico, $\mathrm{n}(\%)$ & $1(4.2)$ & $2(5.6)$ & 0.7193 \\
\hline Trastorno renal hidroelectrolítico, $\mathrm{n}(\%)$ & $5(20.8)$ & $2(5.6)$ & 0.1656 \\
\hline ISO profunda, $\mathrm{n}(\%)$ & $1(4.2)$ & $1(2.8)$ & 0.6626 \\
\hline Consolidación clínica en meses $X \pm \mathrm{DE}$ & $3.7 \pm 0.9$ & $3.9 \pm 1.7$ & 0.6944 \\
\hline Consolidación radiográfica en meses $X \pm \mathrm{DE}$ & $6.3 \pm 2.6$ & $2.7 \pm 0.6$ & 0.0644 \\
\hline Funcionalidad previa, Me [RI] & $17.5[17.5-18]$ & $17[17-18]$ & 0.9934 \\
\hline Funcionalidad actual, Me [RI] & $14[14-17.5]$ & $16[14.5-17.5]$ & 0.6387 \\
\hline Cut Out, $\mathrm{n}(\%)$ & $2(8.3)$ & $0(0.0)$ & 0.3065 \\
\hline Mal posición, $\mathrm{n}(\%)$ & $1(4.2)$ & $0(0.0)$ & 0.8299 \\
\hline Dolor anterior, $\mathrm{n}(\%)$ & $5(20.8)$ & $4(11.1)$ & 0.5078 \\
\hline Seguimiento (días), Me [RI] & $625[330-1110]$ & $765[390-810]$ & 0.4546 \\
\hline Mortalidad, $\mathrm{n}(\%)$ & $5(20.8)$ & $9(25.0)$ & 0.9479 \\
\hline Tiempo de mortalidad en días, Me [RI] & $120[75-705]$ & $630[120-765]$ & 0.3114 \\
\hline
\end{tabular}

veland varían en dispositivos de doble tornillo o perno, limitando la zona de seguridad por el diseño. Lo anterior es una teoría propuesta en Schmith y Teague previamente (2).

El dolor anterior del muslo se presentó frecuentemente pero no debe ser en relación con la longitud del clavo, concluimos que se debe al sitio de inserción más posterior en el trocánter mayor y no tanto a la relación de longitud del clavo.

El seguimiento promedio fue el esperado, con una tasa de mortalidad promedio si lo comparamos con estudios como el de Herrera (9) o Nikkel (8), con tasas aproximadas de $18 \%$ a los 12 meses. La mayoría de pacientes que fallecieron presentaron una causa de mortalidad tardía y la principal causa fue neumonía, la cual está relacionada con la limitación de la movilidad y estasis.

La técnica de enclavijamiento cefalomedular es segura y eficaz para el manejo de frac- turas inestables de cadera extracapsular. En la serie el tiempo quirúrgico y el sangrado fueron escasos y las complicaciones mínimas comparadas con el retorno a la funcionalidad del paciente. El clavo Targon-PF presentó más complicaciones de tipo ortopédico inherentes al implante, así como mayor tiempo quirúrgico. El estudio es en cierta medida limitado, ya que se dio por conveniencia, razón por la cual no es extrapolable a la población de la ciudad. No fue posible realizar una aleatorización del implante sistematizada y la muestra no es representativa del nivel nacional.

Se establecieron diferencias en los resultados funcionales entre los implantes estudiados, con lo cual se abre paso a futuras investigaciones en la biomecánica de los mismos. Al detectar las complicaciones más frecuentes se recomiendan crear y aplicar estrategias con énfasis a minimizarlas, especialmente, la prevención en la formación de escaras, el adecuado control de catéteres y el buen manejo hidroelectrolítico. 
ISSN: 2215-7840, 6(2), julio-diciembre 2015, Fonseca-Caro John Fredy, Matson-Carballo Gustavo, Pereira-Betancourt Carlos, Rivera-Martínez Edgardo

\section{CONCLUSIÓN}

No se observaron diferencias significativas en los resultados clínicos con el uso de clavos Targon-PF y hoja espiral TFN.
CONFLICTOS DE INTERESES: ninguno que declarar.

FINANCIACIÓN: recursos propios de los autores.

\section{REFERENCIAS BIBLIOGRÁFICAS}

1. Bucholtz R, Heckman J, editors. Rockwood and green's fractures in adults, $5^{\text {th }}$ ed. Boston: Lippincott Willian and Wilkins; 2007.

2. Schmith A, Teague D, Callaghan J. Orthopaedic knowledge update, $4^{\text {th }}$ ed. Illinois: American Academy of Orthopaedic Surgeons; 2010.

3. Miyamoto R, Kaplan K, Levine B, Egol K, Zuckerman J. Surgical management of hip fractures: an evidence-based review of the literature. I: Femoral Neck Fractures. J Am Acad Orthop Surg. 2008;16(10):596-607.

4. Lindskog D, Baumgaertner M. Unstable intertrochanteric hip fractures in the elderly. J Am Acad Orthop Surg. 2004; 12(3):179-190

5. Martinez C, Escobar G (ed). Proyecciones nacionales de población, Colombia 1950-2025. Bogotá D.C. Colombia. DANE 1991:1-73.

6. Othman Y. Assessment of a newly designed dynamic condylar screw with an angle of $102^{\circ}$ in treatment of unstable intertrochanteric fractures. Eur J Orthop Surg Traumatol. 2004;14: 225229.

7. Nurmi I, Narinem A, Luthje P, Taninem S. Functional outcome and survival after hip fracture in elderly: a prospective study of 106 consecutive patients. J Orthopaed Traumatol. 2004; 4: 7-14

8. Nikkel L, Edward J, Black K. Impact of comorbidities on hospitalization cost following hip fracture. J Bone Joint Surg Am.2012;94(1):9-17.

9. Herrera J, Martinez A. Results of osteosynthesis with the ITST nail in Fractures of trochanteric region of the femur. Int Orthop 2008; 32(6):767-772.

10. Kevin J. Pugh, M. Intracapsullar hip fractures. Jeffrey S. editor Orthopaedic Knowledge Update 9. Illinois: American Academy of Orthopaedics Surgeons;2008. 399-411

11. Kenneth J. Koval A, Zuckerman,J. Hip fractures: II. Evaluation and treatment of intertrochanteric fractures. J Am Acad Orthop Surg. 1994;2:150-156.

12. Kyle RF, Cabanela ME, Russell TA, Swiontkowski MF, Winquist RA, Zuckerman JD, Schmidt AH, Koval KJ. Fractures of the proximal part of the femur. Instr Course Lect. 1995;44:227-253.

13. O'Neil F Condon F, McGloughlim T, Lenehan B, Coffey J, Walsh M. Dynamic hip screw versus DHS blade a biomechanical comparison of the fixation achieved by each implant in bone. J Bone Joint Surg Br. 2011; 93(5):616-621.

14. Evans E. The treatment of trochanteric fractures of the femur. J Bone Joint Surg Br. 1949; 31(2): 190-203.

15. Miyamoto R, Kaplan K, Levine B, Egol K, Zuckerman J. Surgical management of hip fractures: an evidence-based review of the literature. II: Intertrochanteric Fractures. J Am Acad Orthop Surg 2008;16(11):665-673.

16. Harrington K. The use of methylemethacryl as an adjunct in the internal fixation of unstable comminuted intertronchanteric fractures in osteoportoic patients. J Bone and Joint Surg. 1975;57:744-750.

17. Bridle S, Patel A, Bircher M. Fixation of intertrochanteric fractures of the fémur: A randomised prospective comparison of the gamma nail and the dynamic hip screw. J Bone Joint Surg Br. $1991 ; 73: 330-334$.

18. Adams C, Robinson C, Court-Brown C, McQueen M. Prospective randomized controlled trial of an intramedullary nail versus dynamic screw and plate for intertrochanteric fractures of the fémur. J Orthop Trauma. 2001;15(6):394-400.

19. Pajarinen J, Lindahl J, Michelsson O, Savolainen V, Hirvensalo E. Pertrochanteric femoral fractures treated with a dynamic hip screw or a proximal femoral nail: A randomised study comparing post-operative rehabilitation. J Bone Joint Surg Br. 2005;87(1):76-81.

20. Nuber S, Schönweiss T, Rüter A. Stabilisation of unstable trochanteric femoral fractures: Dynamic Hip Screw (DHS) with trochanteric stabilization plate vs. proximal fémur nail (PFN) [German]. Unfallchirur. 2003;106(1):39-47.

21. Bruijn K, Hartog D, Tuinebreijer W. Reliability of predictors for screw Cut out in intertrochanteric hip fractures. J Bone Joint Surg Am. 2012; 94(14):1266-1272.

22. Gary-Villa E, De la Peña O, Solano A. Resultados del tratamiento de fracturas intertrocantericas inestables con clavo cefalomedular bloqueado. Revista Colombiana de Ortopedia y Traumatologia. $2008 ; 22(1): 60-66$. 